\title{
Comprehensive care for chronic obstructive pulmonary disease
}

\author{
Fanny Wai San Ko, Ka Pang Chan, David Shu Cheong Hui
}

SH Ho Research Center in Respiratory Diseases, Department of Medicine and Therapeutics, The Chinese University of Hong Kong, Hong Kong, China

Contributions: (I) Conception and design: FW Ko, DS Hui; (II) Administrative support: None; (III) Provision of study materials or patients: None; (IV) Collection and assembly of data: None; (V) Data analysis and interpretation: None; (VI) Manuscript writing: All authors; (VII) Final approval of manuscript: All authors.

Correspondence to: Prof. David Shu Cheong Hui. Department of Medicine and Therapeutics, The Chinese University of Hong Kong, Hong Kong, China. Email: dschui@cuhk.edu.hk.

\begin{abstract}
Chronic obstructive pulmonary disease (COPD) is a common chronic disease worldwide and incurs heavy utilization of healthcare resources. Many COPD patients have comorbidities and experience exacerbations in the course of the disease. Correct diagnosis and appropriate disease assessment are essential for clinical management. Comprehensive care for patients with different severity of disease aims to offer personalized treatment to suit individual needs. Patients with recent exacerbations also need extra care for the post-acute and rehabilitation phases. Comprehensive care consists of self-management and pulmonary rehabilitation and involves multiple healthcare providers working together closely to provide formal structured programmes for patients. The setting, professionals involved, content and the duration of programme vary a lot among different comprehensive care models. Some randomized controlled trials suggested there was improvement in quality of life, exercise capacity and reduced hospital admissions for participants in comprehensive care programmes compared with controls. However, other studies showed that such programmes might not confer benefits and might even bring harm. The reason for the differences in clinical effect of programmes might be due to differences in study design, components and subjects involved in the studies. Careful evaluation of each programme is thus mandatory. Further research is needed to evaluate the safety and effectiveness of comprehensive care management for COPD patients, both at the stable and post-acute exacerbation state.
\end{abstract}

Keywords: Comprehensive care; chronic obstructive pulmonary disease (COPD); self-management; pulmonary rehabilitation

Submitted Jul 19, 2019. Accepted for publication Sep 27, 2019.

doi: $10.21037 /$ jtd.2019.09.81

View this article at: http://dx.doi.org/10.21037/jtd.2019.09.81

Chronic obstructive pulmonary disease (COPD) is a common chronic disease globally. Patients who suffer from COPD have significant morbidity, and thus incur heavy utilization of healthcare resources (1-3). Many COPD patients experience exacerbations in the course of the disease (4). COPD exacerbations are defined as an acute worsening of respiratory symptoms that results in additional therapy (1). The cost of hospitalizations for COPD exacerbations is marked and is the single largest source of healthcare expenditure for these patients $(5,6)$. Frequent exacerbations have an adverse effect on both the quality of life (QOL) and pulmonary function of COPD patients (7-9). In addition, many patients are elderly with multiple comorbidities, such as cardiac disease, diabetes mellitus, hypertension, osteoporosis and psychological disorders $(10,11)$. Correct diagnosis and appropriate disease assessment are essential for clinical management of all COPD patients.

Comprehensive care for patients aims to offer personalized treatment to suit individual needs for patients with different severity of disease. There is no formal definition for the term "comprehensive care for COPD". 
This probably reflects a "difficulty in conceptualizing a complex and changing process aimed at addressing the particular problems of individuals with chronic illnesses" $(12,13)$.

Overall, comprehensive care is to provide personal health services for diagnosis, treatment, follow-up and rehabilitation of patients. Models of care need to cater for both the need of the patients and the healthcare structure. In this article, we will describe different components and integration of these components.

\section{Components of "comprehensive care"}

Components of comprehensive care vary in different programmes. In Wagg's model of spectrum of support for COPD, the elements of action plan, education, selfmanagement, pulmonary rehabilitation and integrated care are along a continuum of care with each of the more complex care encompassing the components with less complexity (14). Comprehensive care involves interdisciplinary, patient-centered and holistic approach to the management of complex COPD patients (13).

\section{Self-management}

Self-management is defined as "an individual's ability to detect and manage symptoms, treatment, physical and psychosocial consequences, and lifestyle changes inherent in living with a chronic condition" (15). Interventions are often multi-component and should be structured but personalized. Interventions aims at motivating, engaging and supporting the patients to positively adapt their health behaviour(s) that patients can develop skills to better selfmanage their disease (16).

Components of self-management have a lot of combinations with different modes, location, professions involved and topics cover. In addition, the duration, presence of action plan, exercise and behavioural components also varied among different programmes. Optimal use of inhalers (which is highly specific to airway diseases; much like insulin injections being specific for patients with diabetes) is an important part of self-management and that involves health coaching, skills training and competency, learning at home (e.g., online instructional videos) and regular review. Examples of components are shown in Table 1. The length of a programme can range from hours to months and it can be based in different locations or combination of locations, e.g., home-based together with clinic-based. The programme can be in groups or as individual lessons. The content of the topics covered can be diverse in different programmes. There is no information regarding the best way to offer self-management to patients (17). An example of the programme by McGeoch et al. (18) is that patients in the active arm received structured education (an individual 1-hour session delivered by a practice nurse or respiratory educator in association with their general practitioner). The structured education included methods of early recognition of COPD exacerbations and a range of appropriate selfinitiated interventions including antibiotics and short-course oral corticosteroids. Patients were also given instructions to make early contact with their general practice during exacerbations.

A study using online Delphi surveys to assess the relevance and feasibility of predetermined self-management behaviours, found that focus on smoking cessation, avoiding stimuli, physical activity/exercise, influenza vaccination, adherence to pharmacotherapy, managing stress and anxiety, awareness for recurrent exacerbations, early detection of symptom deterioration and medical treatment of exacerbations are considered important. This study also suggested that when the patient had a change in condition, from stable phase, to mild deterioration, exacerbation, recovery and back to stable phase, the change in condition would require aggregating specific self-management behaviours from the patient to maximally reduce the harmful effect of exacerbations (19).

In a review by Zwerink et al. in 2014 that involved 23 studies with 3,189 participants comparing selfmanagement versus usual care, it was found that selfmanagement interventions in patients with COPD were associated with improved health-related QOL as measured by the St Georges Respiratory Questionnaire (SGRQ), a reduction in respiratory-related and all cause hospital admissions, and improvement in dyspnoea measured by the Modified Medical Research Council Dyspnea Scale (mMRC). There was, however, no significant improvement in exercise capacity as measured by 6-min walking test and mortality. Subgroup analyses depending on the use of an exercise programme as part of the intervention revealed no statistically significant differences between studies with and without exercise programmes when using the primary outcomes of health-related QOL and respiratory-related hospital admissions. As action plans were included in most studies in the meta-analysis, subgroup analysis could not be performed to assess whether presence of action plan would be better than none in this review (17). 
Table 1 Examples of components of self-management programmes (17)

Modes

Face-to-face

Education booklet

Individual teaching

Group teaching

Telephone calls

Home-based

Out-patient clinic

Community-based

Primary care practice

Hospital based (out-patient)

Professionals

Nurse

Respiratory nurse

Respiratory therapist

Respiratory physiotherapist

Exercise practitioner

Physician assistant

Dietician

General practitioner

Respiratory specialist

Case manager

Clinical pharmacist

Graduate student in psychology

Topics

Education regarding the disease

Methods for smoking cessation

Use of medication

Coping with breathlessness

Breathing and coughing techniques

Advice about exercise and activities

Breathing and coughing techniques

Energy conservation during day-by-day activities

Relaxation exercises

Understanding and using plan of action for acute exacerbations

Adopting a healthy lifestyle

Table 1 (continued)
Table 1 (continued)

\author{
Leisure activities and travelling \\ Simple home exercise programme \\ Long-term oxygen therapy when appropriate \\ Advice about nutrition \\ Stress management \\ Duration (Variable, some examples) \\ One group session of two hours, three individual sessions of \\ $40 \mathrm{~min}$, and one to 10 sessions of $20 \mathrm{~min}$ at home
}

Two to three sessions of two to three hours, four phone calls; exercise: daily, 30 to $60 \mathrm{~min}$

At least 10 educational calls 10 to 15 min each, at least one face-to-face $45 \mathrm{~min}$

Six monthly individual sessions of at least $30 \mathrm{~min}$

Exercise programme/action plan

In some programmes

Cognitive-behavioural therapy

In some programmes, examples: cognitive-behavioural therapy, goal setting, providing feedback to the participant

Different programme have different combinations.

In a review by Lenferink et al. that compared the effectiveness of COPD self-management with action plans for acute exacerbations of COPD with usual care, which involved 22 randomised controlled trials (RCTs) with 3854 participants, it was found that the active group had improvements in health-related QOL as measured with the SGRQ, and had a lower probability of respiratory-related hospital admissions versus the control group. No excess allcause mortality risk was observed. However, exploratory analysis showed a small, but significantly higher respiratoryrelated mortality rate for self-management compared to usual care (20). This may be partly explained by the use of COPD-specific action plans for patients with COPD and comorbidities (21). When developing a self-management program, there should be audits to ensure safety and effectiveness for participating patients.

Apart from improvement in QOL and less respiratory hospital admission, self-management programme was associated with reducing the length of exacerbations and decreasing cost. In a study by Trappenburg et al. that involved 233 subjects with COPD randomized to receive an individualised action plan to recognise and anticipate 
early exacerbation symptoms or usual care, the active arm had positive effects on exacerbation by reducing the length of exacerbation and accelerating recovery time (22). Effing et al. randomized COPD patients to four 2-h selfmanagement sessions, with or without training in selftreatment of exacerbations, found that those with training in self-treatment of exacerbations had fewer exacerbation days and lower costs. An average of $€ 154$ was saved for each patient by self-treatment and the numbers needed to treat to prevent one hospitalisation and one health care contact were 7.4 and 0.9 respectively. This was, however, associated with a significantly higher reported use of courses of prednisolone and antibiotics in the self-treatment group (23).

On the other hand, not all studies found positive effects with self-management. A study from the Netherlands randomized 248 moderately severe COPD patients, who were receiving close to optimal treatment, to a selfmanagement and a control group. The self-management intervention consisted of a skill-oriented patient education programme and a near-home fitness programme, on top of usual care. No differences in the SGRQ scores and 6-min walking distance were found between the groups at 1 year. In addition, the intervention group reported more exacerbations than the control group, with about $70 \%$ of these exacerbations being self-treated at home (24). While each programme had different patient characteristics and components of intervention and duration, it is important to evaluate each programme for the efficacy and safety, in particular in patients with more severe COPD who may have more comorbidities and higher risk of having exacerbations.

Components of self-management consist of a lot of combinations such as different modes, location, professions involved, topics cover, presence of action plan, with also a variety of exercise and behavioural components offered. Without standardization, the safety and effectiveness of new programmes must be monitored with care to ensure safety and efficacy.

\section{Pulmonary rebabilitation}

Pulmonary rehabilitation has established itself as an important component of an integrated care model in the management of COPD. It is defined as "a comprehensive intervention based on a thorough patient assessment followed by patient-tailored therapies, which include, but are not limited to, exercise training, education, and behavior change, designed to improve the physical and psychological condition of people with chronic respiratory disease and to promote the long-term adherence of health-enhancing behaviors." (25) Pulmonary rehabilitation encompasses self-education but adds more components to it. A vital part of pulmonary rehabilitation is structured exercise training. A complex combination of functional, metabolic and anatomical alterations results in suboptimal muscle performance in COPD patients (26). Exercise training can improve muscle function and this leads to gain in exercise capacity despite no significant changes in lung function $(27,28)$. Improvements in exercise performance and muscle function are proportionally larger than increases in fat-free mass (27).

There are many different types of exercise training, including endurance training, interval training, resistance training, upper limb training, flexibility training, neuromuscular electrical stimulation and respiratory muscle training. Training needs to be individualized. Before the start of a training program, an exercise assessment is required for planning of the exercise prescription. Some patients may need supplemental oxygen during exercise. Patients with significant comorbidities such as cardiovascular comorbidities may need caution in training to ensure safety (25).

Pulmonary rehabilitation is a multidisciplinary intervention. A 'typical' multidisciplinary rehabilitation team consists of a chest physician with rehabilitation expertise, a physiotherapist and/or exercise training specialist, a nutritional expert, a psychologist, a social worker, an occupational therapist and a nurse (29). Clear guidance as to the optimal or minimal duration of the programme, location, type of patients and essential components, etc. is largely missing.

The American Thoracic Society/European Respiratory Society and American College of Chest Physicians/ American Association of Cardiovascular and Pulmonary Rehabilitation guidelines on pulmonary rehabilitation recommend progressive high-intensity physical exercises, such as treadmill walking, ergometry cycling, strengthening exercises for muscle groups of the upper and lower limbs, and unsupported arm exercises at least three times a week for 6-12 weeks $(30,31)$. Self-management and educational programmes could have additional positive effects on the wellbeing of patients with COPD (31). However, the impact of psychosocial interventions and nutritional/hormonal supplements remain uncertain.

Pulmonary rehabilitation is suitable for both patients at stable state and post-exacerbation. For patient in stable 
state, pulmonary rehabilitation is beneficial to patients with both advanced/severe and less advanced/less severe COPD. In a review by McCarthy et al., involving 65 RCTs with 3,822 participants, mostly stable COPD subjects, pulmonary rehabilitation programmes, with exercise training for at least four weeks with or without education and/or psychological support, relieves fatigue and dyspnoea, improves health-related QOL, emotional function, the sense of control that individuals have over their condition and exercise capacity when compared with the controls (32). Previously, most pulmonary rehabilitation programs enrolled individuals with more advanced COPD (33). More recent data suggest that patients with less severe disease also benefit from pulmonary rehabilitation $(34,35)$. In a RCT in the Netherlands with community-based pulmonary rehabilitation for patients with less advanced airflow obstruction (FEV1 about $60 \%$ predicted), an intensive 4-month rehabilitation resulted in significant improvements in breathlessness, exercise performance, health-related QOL, muscle strength and body composition. When patients participated in an active maintenance programme in the following 20 months, the QOL, breathlessness and functional exercise capacity remained significantly better in the intervention group over an evaluation period of 2 years (35).

Previous studies have also shown that pulmonary rehabilitation post-acute exacerbation is safe (36). Early pulmonary rehabilitation has moderate to large effects on health-related QOL and exercise capacity in patients with COPD after an exacerbation, however there was heterogeneity of effects on mortality and hospital readmissions (37). The European Respiratory Society statement by Wedzicha et al. in 2016 made a conditional recommendation against the initiation of pulmonary rehabilitation during hospitalisation and a conditional recommendation in favour of starting pulmonary rehabilitation $\leq 3$ weeks after hospital discharge (38). Our previous RCT on early pulmonary rehabilitation with exercise training for 2 months (thrice per week for 8 weeks) after acute exacerbation of COPD found that an early pulmonary rehabilitation led to improvement in QOL up to 6 months, but did not lead to a reduction in health-care utilization including readmission, COPD exacerbation rates and emergency department visits, at 1 year (39). The rehabilitation programme for the active group started 2-3 weeks after discharge from hospital. A dedicated physiotherapist conducted the exercise training sessions with supervised training on-arm and leg weight handling, use of treadmill, and arm cycling. The training intensity, based on the tolerability and physiological variables. For patient who could complete a baseline cardiopulmonary exercise (CPE) test, the target training intensity was $60-70 \%$ of maximal oxygen uptake $\left(\mathrm{VO}_{2} \mathrm{Max}\right)$ of the baseline CPE. For patients who were unable to do not complete the $\mathrm{CPE}$ assessment an exercise intensity to achieve $60-70 \%$ of their maximum predicted heart rate was used as the target. Patients were also advised to perform at least $20 \mathrm{~min}$ of home exercises a day. Techniques on proper breathing techniques and how to cope with daily activities were also taught. We found that the St George's respiratory questionnaire total score was lower in the intervention group compared to the control group $(40.15 \pm 19.10 \mathrm{vs}$. $46.91 \pm 18.21, \mathrm{P}=0.01$ and $42.3 \pm 20.06$ vs. $51.44 \pm 18.98$, $\mathrm{P}=0.01$ at 3 and 6 months respectively). The intervention group and control group demonstrated a $53.3 \%$ and $43.3 \%$ risk of readmissions at 12 months (incident risk ratio 0.97 , 95\% CI: $0.57-1.60, \mathrm{P}=0.90)$. We found that many patients (54 out of the 210 patients approached for recruitment) could not join the study due to 'unlikely to benefit me', "clashed with other social activities", or "nobody to accompany them". Patient motivation and family support were challenges for the programme.

\section{Integrated/comprehensive care}

The World Health Organization defines "integrated care" as "a concept bringing together inputs, delivery, management and organization of services related to diagnosis, treatment, care, rehabilitation and health promotion" (40). In contrast, integrated healthcare services delivery is defined as "an approach to strengthen people-centered health systems through the promotion of the comprehensive delivery of quality services across the life-course, designed according to the multidimensional needs of the population and the individual and delivered by a coordinated multidisciplinary team of providers working across settings and levels of care. It should be effectively managed to ensure optimal outcomes and the appropriate use of resources based on the best available evidence, with feedback loops to continuously improve performance and to tackle upstream causes of ill health and to promote well-being through intersectoral and multisectoral actions" (41). Nici et al. described 2 key aspects of integrated care for COPD: (I) providing the right care for the right patient at the right time; (II) providing a seamless transition (coordination) of care. For COPD patient management, patients would need a whole range of services from a multidisciplinary team (12). 
In integrated care, multiple healthcare providers work together closely to provide formal structured programmes for COPD patients. In the review by Kruis et al. that included RCTs (26 trials with 2,997 people, with a follow-up ranging from 3 to 24 months in 11 countries, with different composition in terms of health care professions and service provided as illustrated in Table 2, evaluating integrated disease management programs in people with COPD, integrated care not only improved QOL and exercise capacity, but also reduced hospital admissions and hospital days per person (42). Many of these programmes have incorporated components of selfmanagement and exercise/pulmonary rehabilitation into them. Pulmonary rehabilitation may include interventions for exercise training, education and behavior change. Some comprehensive programme may include smoking cessation, medication management, nutrition, follow up and communication with a multidisciplinary team (42). In the COPD Patient Management European Trial (COMET), a multicenter (with 33 centers in France, Germany, Italy and Spain) RCT to investigate the effectiveness of a multicomponent home-based COPD disease management intervention specifically adapted for patients with severe COPD (FEV $1<50 \%$ ), it was found that the active programme with a self-management program, home monitoring and an e-health telephone/web platform did not significantly reduce unplanned all-cause hospitalisation days, but reduced acute care hospitalisation days and mortality in severe COPD patients when compared to the control group (43).

The setting, professionals involved, content and duration of programme varied a lot among different comprehensive care models. There is no information regarding what combination of components can offer improved outcome. It is thus impossible to comment on the completion rates for the individual aspects of comprehensive care and their specific success rates. Not all comprehensive care models are beneficial to patients. A 2-year cluster RCT in 40 general practices in the Netherlands found that integrated care programme for patients with COPD in primary care increased costs but did not improve health outcomes in QOL and COPD exacerbations except with a self-reported higher degree of daily activities $(44,45)$. In another RCT comparing a comprehensive care management program with guideline-based usual care in COPD patients who had been hospitalized in the past year, patients in the intervention group were provided with COPD education during
1 group session and 4 individual sessions, an action plan for identification and treatment of COPD exacerbations, and scheduled proactive telephone calls for case management. Patients in both the usual care and intervention groups were given a COPD informational booklet; a copy of COPD guidelines was distributed to their primary care providers and their primary care providers were advised to manage their patients according to these guidelines. The study was stopped prematurely as the intervention group had higher unanticipated excess mortality and there was also no decrease in COPD-related hospitalizations (21).

Telemedicine interventions may be a potentially useful component in a comprehensive model. However, studies have shown uncertainties whether these can improve QOL and psychological outcomes of COPD patients. The costs of home-based telemedicine programs varied substantially by program components, disease type, equipment used and services provided (46-48) and more studies are needed to assess the cost-effectiveness.

In patients who were recently admitted for AECOPD, a recent RCT has shown that patients in the comprehensive programme, compared with those treated with usual care, had decrease the hospital readmission rate at 1-year follow-up. In addition, the dyspnea score measured by MMRC and QOL measured by SGRQ score also showed improvement. The intervention group received a comprehensive, individualised care plan which included education provided by a respiratory nurse (two $1-\mathrm{h}$ sessions of individual education sessions including smoking cessation, pathophysiology of COPD, anatomy and physiology of the respiratory system, dyspnoea management, technique of using medications, selfmanagement and exacerbation-reduction skills, nutrition, social and community support, coping with psychological distress and relaxation techniques, and, if appropriate, knowledge on long-term oxygen therapy), pulmonary rehabilitation support provided by a physiotherapist (education on energy-conservation techniques, breathing and sputum-removal techniques and exercise benefits and strategies. Each patient was provided with an individualised physical training programme to perform at home or a short course of outpatient pulmonary rehabilitation), 3-monthly respiratory nurse telephone calls over 1 year, and followup by a respiratory specialist at the clinic once every 3 months for 1 year. If necessary, ad hoc arrangements to see a respiratory physician as an outpatient could be made (49).

As in self-management and pulmonary rehabilitation, 
Table 2 Different Integrated care programme has different composition in terms of health care professions and service provided. Below is a list of some components involved in previous studies as examples (42)

\begin{tabular}{|c|}
\hline Settings \\
\hline Home \\
\hline Primary care \\
\hline Secondary care \\
\hline Hospital based home care programme \\
\hline Professionals \\
\hline Respiratory physicians \\
\hline Respiratory nurses \\
\hline Registered nurse case managers \\
\hline Physiotherapist \\
\hline Occupational therapist \\
\hline Dietician \\
\hline Psychologist \\
\hline Social worker \\
\hline Primary care doctor \\
\hline Hospital medical officer \\
\hline Pharmacist \\
\hline Service \\
\hline Developed care plans \\
\hline Worked with protocols \\
\hline Provided education to patients \\
\hline Education to carers \\
\hline Classes \\
\hline Booklets \\
\hline Smoking cessation \\
\hline Exercise training \\
\hline Breathing retraining \\
\hline Evacuation of mucus \\
\hline Chest wall and abdominal muscle wall work \\
\hline Intensive rehabilitation program \\
\hline Relaxation techniques \\
\hline Recreational activities. \\
\hline Occupational therapy \\
\hline Tailored self-management of the disease \\
\hline
\end{tabular}

Table 2 (continued)
Table 2 (continued)

Teaching of self-management skills

Assessing psychological and spiritual needs.

Action plan

Exacerbation management

Ambulatory oxygen assessment

Supervision of domiciliary oxygen

Nebuliser assessments

Review of medication

Inhalation techniques

Monitoring of treatment compliance

Optimizing pharmacotherapy treatment

Advice about nutrition

Advice about exercise

Introduction to a patient support group

Telephone calls

Home visits

Annual influenza vaccination

Monitoring of treatment compliance

Duration

Variable, examples: 12 weeks, 4 months, 6 months, 18 months, 24 months

each integrated/comprehensive programme care would need evaluation to assess if it would confer any benefit to the patients.

\section{Integration}

Since there is no formal definition for the term 'comprehensive care for COPD' and there is no reference model for the design, programme contents (including components, person/professions involved, targeted patient groups) should be planned according to the resources available, health care system infrastructure and patient needs. We propose that comprehensive care is a programme with integration of self-management, pulmonary rehabilitation and integrated care. The model would require a multidisciplinary team of professionals to offer care to patients from diagnosis, disease assessment, to a variety of services which suit the patients' needs and disease severity 


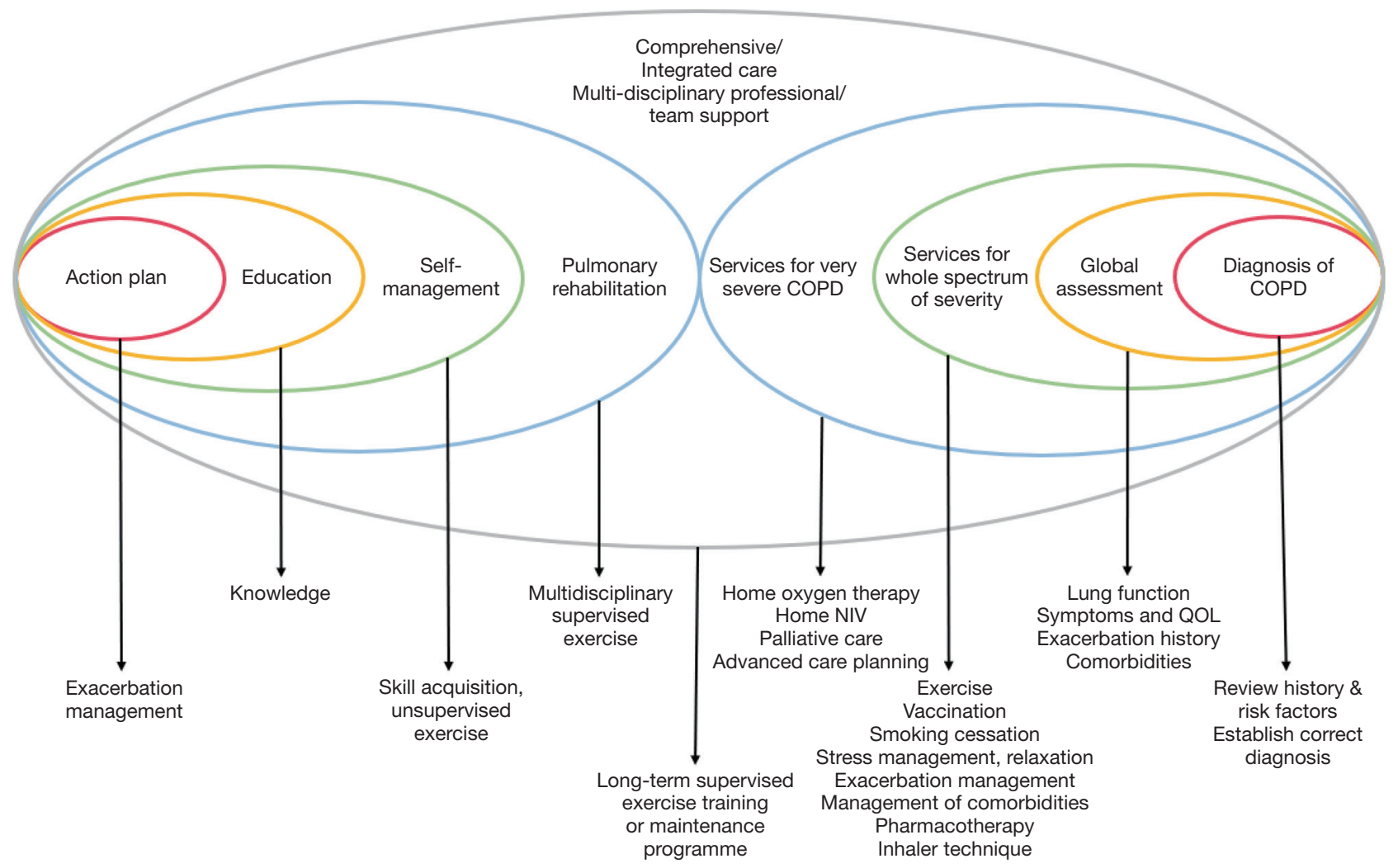

Figure 1 Illustration of comprehensive/integrated care model. Comprehensive care includes action plan, education, self-management and pulmonary rehabilitation provided a mutlidisciplinary team in a coordinated way. Components were added on top of the model of Wagg et al. (14).

as illustrated in Figure 1 [with components added on top of the model of Wagg et al. (14)].

\section{Conclusions}

Comprehensive care for COPD patients involves a variety of services tailored to individual needs. As COPD patients are heterogeneous in terms of their severity, exacerbations, comorbidities and social needs, different programmes may be needed to cater for their needs. Mild patients may benefit from self-management and pulmonary rehabilitation. More severe patients may need more intensive support such as comprehensive care. Very severe patients may require additional support for home non-invasive ventilation (50), palliative care (51) and advanced care planning (52) built into the model. Patients post-acute exacerbation may need extra support. Suggestions for some components of the programme are shown in Figure 1. As some of the models tested previously cannot provide benefit to patients and may even do harm, auditing of the model with continuous improvement would be necessary. More studies are required in order to assess the timing of commencement of the programme, duration of care, professionals involved, composition and topics/areas covered when one decides his/her own local COPD wellness program. With advances in technology, the model of care may also be delivered with telemedicine/telemonitoring. More research is needed to ensure safety and effectiveness of comprehensive care management for COPD patients, both at the stable and post-acute exacerbation state.

\section{Acknowledgments}

None.

\section{Footnote}

Conflicts of Interest: The authors have no conflicts of interest 
to declare.

Ethical Statement: The authors are accountable for all aspects of the work in ensuring that questions related to the accuracy or integrity of any part of the work are appropriately investigated and resolved.

\section{References}

1. Global Strategy for the Diagnosis, Management and Prevention of COPD, Global Initiative for Chronic Obstructive Lung Disease (GOLD) 2019. Accessed 02 Jul 2019. Available online: https://goldcopd.org/gold-reports/

2. Buist AS, McBurnie MA, Vollmer WM, et al. International variation in the prevalence of COPD (the BOLD Study): a population-based prevalence study. Lancet 2007;370:741-50.

3. Ko FW, Hui DS, Lai CK. Worldwide burden of COPD in high- and low-income countries. Part III. Asia-Pacific studies. Int J Tuberc Lung Dis 2008;12:713-7.

4. Ko FW, Chan KP, Hui DS, et al. Acute exacerbation of COPD. Respirology 2016;21:1152-65.

5. Toy EL, Gallagher KF, Stanley EL, et al. The economic impact of exacerbations of chronic obstructive pulmonary disease and exacerbation definition: a review. COPD 2010;7:214-28.

6. Britton M. The burden of COPD in the U.K.: results from the Confronting COPD survey. Respir Med 2003;97 Suppl C:S71-9.

7. Seemungal TA, Donaldson GC, Paul EA, et al. Effect of exacerbation on quality of life in patients with chronic obstructive pulmonary disease. Am J Respir Crit Care Med 1998;157:1418-22.

8. Donaldson GC, Seemungal TA, Bhowmik A, et al. Relationship between exacerbation frequency and lung function decline in chronic obstructive pulmonary disease. Thorax 2002;57:847-52.

9. Anzueto A, Leimer I, Kesten S. Impact of frequency of COPD exacerbations on pulmonary function, health status and clinical outcomes. Int J Chron Obstruct Pulmon Dis 2009;4:245-51.

10. Chatila WM, Thomashow BM, Minai OA, et al. Comorbidities in chronic obstructive pulmonary disease. Proc Am Thorac Soc 2008;5:549-55.

11. Mannino DM, Higuchi K, Yu TC, et al. Economic Burden of COPD in the Presence of Comorbidities. Chest 2015;148:138-50.

12. Nici L, ZuWallack R. An official American Thoracic
Society workshop report: the Integrated Care of The COPD Patient. Proc Am Thorac Soc 2012;9:9-18.

13. Nici L, ZuWallack R. Integrated Care in Chronic Obstructive Pulmonary Disease and Rehabilitation. COPD 2018;15:223-30.

14. Wagg K. Unravelling self-management for COPD: what next? Chron Respir Dis 2012;9:5-7.

15. Barlow J, Wright C, Sheasby J, et al. Self-management approaches for people with chronic conditions: a review. Patient Educ Couns 2002;48:177-87.

16. Effing TW, Vercoulen JH, Bourbeau J, et al. Definition of a COPD self-management intervention: International Expert Group consensus. Eur Respir J 2016;48:46-54.

17. Zwerink M, Brusse-Keizer M, van der Valk PD, et al. Self management for patients with chronic obstructive pulmonary disease. Cochrane Database Syst Rev 2014;3:CD002990.

18. McGeoch GR, Willsman KJ, Dowson CA, et al. Selfmanagement plans in the primary care of patients with chronic obstructive pulmonary disease. Respirology 2006;11:611-8.

19. Korpershoek YJ, Bruins Slot JC, Effing TW, et al. Selfmanagement behaviors to reduce exacerbation impact in COPD patients: a Delphi study. Int J Chron Obstruct Pulmon Dis 2017;12:2735-46.

20. Lenferink A, Brusse-Keizer M, van der Valk PD, et al. Self-management interventions including action plans for exacerbations versus usual care in patients with chronic obstructive pulmonary disease. Cochrane Database Syst Rev 2017;8:CD011682.

21. Fan VS, Gaziano JM, Lew R, et al. A comprehensive care management program to prevent chronic obstructive pulmonary disease hospitalizations: a randomized, controlled trial. Ann Intern Med 2012;156:673-83.

22. Trappenburg JC, Monninkhof EM, Bourbeau J, et al. Effect of an action plan with ongoing support by a case manager on exacerbation-related outcome in patients with COPD: a multicentre randomised controlled trial. Thorax 2011;66:977-84.

23. Effing T, Kerstjens H, van der Valk P, et al. (Cost)effectiveness of self-treatment of exacerbations on the severity of exacerbations in patients with COPD: the COPE II study. Thorax 2009;64:956-62.

24. Monninkhof E, van der Valk P, van der Palen J, et al. Effects of a comprehensive self-management programme in patients with chronic obstructive pulmonary disease. Eur Respir J 2003;22:815-20.

25. Spruit MA, Singh SJ, Garvey C, et al. An official American 
Thoracic Society/European Respiratory Society statement: key concepts and advances in pulmonary rehabilitation.

Am J Respir Crit Care Med 2013;188:e13-64.

26. Jaitovich A, Barreiro E. Skeletal Muscle Dysfunction in Chronic Obstructive Pulmonary Disease. What We Know and Can Do for Our Patients. Am J Respir Crit Care Med 2018;198:175-86.

27. Franssen FM, Broekhuizen R, Janssen PP, et al. Effects of whole-body exercise training on body composition and functional capacity in normal-weight patients with COPD. Chest 2004;125:2021-8.

28. Spruit MA, Gosselink R, Troosters T, et al. Resistance versus endurance training in patients with COPD and peripheral muscle weakness. Eur Respir J 2002;19:1072-8.

29. Troosters T, Blondeel A, Janssens W, et al. The past, present and future of pulmonary rehabilitation. Respirology. 2019;24:830-7.

30. Nici L, Donner C, Wouters E, et al. American Thoracic Society/European Respiratory Society statement on pulmonary rehabilitation. Am J Respir Crit Care Med 2006;173:1390-413.

31. Ries AL, Bauldoff GS, Carlin BW, et al. Pulmonary Rehabilitation: Joint ACCP/AACVPR Evidence-Based Clinical Practice Guidelines. Chest 2007;131:4S-42S.

32. McCarthy B, Casey D, Devane D, et al. Pulmonary rehabilitation for chronic obstructive pulmonary disease. Cochrane Database Syst Rev 2015;(2):CD003793.

33. Lacasse Y, Goldstein R, Lasserson TJ, et al. Pulmonary rehabilitation for chronic obstructive pulmonary disease. Cochrane Database Syst Rev 2006;(4):CD003793.

34. Vogiatzis I, Terzis G, Stratakos G, et al. Effect of pulmonary rehabilitation on peripheral muscle fiber remodeling in patients with COPD in GOLD stages II to IV. Chest 2011;140:744-52.

35. van Wetering CR, Hoogendoorn M, Mol SJ, et al. Shortand long-term efficacy of a community-based COPD management programme in less advanced COPD: a randomised controlled trial. Thorax 2010;65:7-13.

36. Puhan MA, Scharplatz M, Troosters T, et al. Respiratory rehabilitation after acute exacerbation of COPD may reduce risk for readmission and mortality -- a systematic review. Respir Res 2005;6:54.

37. Puhan MA, Gimeno-Santos E, Cates CJ, et al. Pulmonary rehabilitation following exacerbations of chronic obstructive pulmonary disease. Cochrane Database Syst Rev 2016;12:CD005305.

38. Wedzicha JA Ers Co-Chair, Miravitlles M, Hurst JR, et al. Management of COPD exacerbations: a European
Respiratory Society/American Thoracic Society guideline. Eur Respir J 2017. doi: 10.1183/13993003.00791-2016.

39. Ko FW, Dai DL, Ngai J, et al. Effect of early pulmonary rehabilitation on health care utilization and health status in patients hospitalized with acute exacerbations of COPD. Respirology 2011;16:617-24.

40. Gröne O, Garcia-Barbero M, WHO European Office for Integrated Health Care Services. Integrated care: a position paper of the WHO European Office for Integrated Health Care Services. Int J Integr Care 2001;1:e21.

41. WHO Regional Office for Europe. Strengthening people-centred health systems in the WHO European Region: framework for action on integrated health services delivery. 2016. Assessed 02 Jul 2019. Available online: http://www.euro.who.int/_data/assets/pdf_ file/0004/315787/66wd15e_FFA_IHSD_160535.pdf?ua=1

42. Kruis AL, Smidt N, Assendelft WJ, et al. Integrated disease management interventions for patients with chronic obstructive pulmonary disease. Cochrane Database Syst Rev 2013;10:CD009437.

43. Kessler R, Casan-Clara P, Koehler D, et al. COMET: a multicomponent home-based disease-management programme versus routine care in severe COPD. Eur Respir J 2018. doi: 10.1183/13993003.01612-2017.

44. Kruis AL, Boland MR, Assendelft WJ, et al. Effectiveness of integrated disease management for primary care chronic obstructive pulmonary disease patients: results of cluster randomised trial. BMJ 2014;349:g5392.

45. Boland MR, Kruis AL, Tsiachristas A, et al. Costeffectiveness of integrated COPD care: the RECODE cluster randomised trial. BMJ open 2015;5:e07284.

46. Gregersen TL, Green A, Frausing E, et al. Do telemedical interventions improve quality of life in patients with COPD? A systematic review. Int J Chron Obstruct Pulmon Dis 2016;11:809-22.

47. Cartwright M, Hirani SP, Rixon L, et al. Effect of telehealth on quality of life and psychological outcomes over 12 months (Whole Systems Demonstrator telehealth questionnaire study): nested study of patient reported outcomes in a pragmatic, cluster randomised controlled trial. BMJ 2013;346:f653.

48. Michaud TL, Zhou J, McCarthy MA, et al. Costs of Home-Based Telemedicine Programs: A Systematic Review. Int J Technol Assess Health Care 2018;34:410-8.

49. Ko FW, Cheung NK, Rainer TH, et al. Comprehensive care programme for patients with chronic obstructive pulmonary disease: a randomised controlled trial. Thorax 
2017;72:122-8.

50. Shah NM, D'Cruz RF, Murphy PB. Update: non-invasive ventilation in chronic obstructive pulmonary disease. J Thorac Dis 2018;10:S71-9.

51. Fusi-Schmidhauser T, Riglietti A, Froggatt K, et al. Palliative Care Provision for Patients with Advanced

Cite this article as: Ko FW, Chan KP, Hui DS. Comprehensive care for chronic obstructive pulmonary disease. J Thorac Dis 2019;11(Suppl 17):S2181-S2191. doi: 10.21037/jtd.2019.09.81
Chronic Obstructive Pulmonary Disease: A Systematic Integrative Literature Review. COPD 2018;15:600-11.

52. Jabbarian LJ, Zwakman M, van der Heide A, et al. Advance care planning for patients with chronic respiratory diseases: a systematic review of preferences and practices. Thorax 2018;73:222-30. 